\title{
Berezinskii-Kosterlitz-Thouless transition in disordered multichannel Luttinger liquids
}

\author{
Max Jones, ${ }^{1}$ Igor V. Lerner, ${ }^{1}$ and Igor V. Yurkevich ${ }^{2,3}$ \\ ${ }^{1}$ School of Physics and Astronomy, University of Birmingham, Birmingham B15 2TT, United Kingdom \\ ${ }^{2}$ Nonlinearity and Complexity Research Group, School of Engineering and Applied Science, \\ Aston University, Birmingham B4 7ET, United Kingdom \\ ${ }^{3}$ Institute of Fundamental and Frontier Sciences, University of Electronic Science and Technology of China, \\ Chengdu 610054, People's Republic of China \\ (Received 22 September 2017; published 27 November 2017)
}

\begin{abstract}
We study the phase transition between conducting and insulating states taking place in disordered multichannel Luttinger liquids with interchannel interactions. We derive renormalization-group equations which are perturbative in disorder but nonperturbative in interaction. In the vicinity of the simultaneous phase transition in all channels, these equations become a set of coupled Berezinskii-Kosterlitz-Thouless equations, which we analyze within two models: an array of identical wires and a two-channel model with distinct channels. We show that a competition between disorder and interaction results in a variety of phases, expected to be observable at intermediate temperatures where the interaction and disorder are relevant but weak hybridization and the charge-density-wave interaction may be ignored.
\end{abstract}

DOI: 10.1103/PhysRevB.96.174210

\section{INTRODUCTION}

The effect of electron-electron interactions on transport properties of disordered systems has attracted a lot of attention since the early prediction [1] and subsequent renormalizationgroup (RG) analysis [2] of the disorder-driven metal-insulator transition. Weak localization corrections to diffusive transport $[2,3]$ are enhanced by the Coulomb interactions, signaling further localization of the system [4]. Interactions in strongly localized systems lead to a metal-insulator transition at a finite temperature proportional to the interaction strength (manybody localization [5]), suggesting that the interaction favors delocalization. Experiments on very clean two-dimensional systems show signatures of a metal-insulator transition driven by a changing of interaction strength (for a review, see Ref. [6]).

A theoretical description of the interaction effects in a generic disordered electron system requires nonperturbative approaches, which are most developed for one-dimensional systems in which interactions can be treated nonperturbatively in terms of the Luttinger liquid (LL) theory [7]. This makes it tempting to tackle transport in higher-dimensional anisotropic disordered strongly correlated systems by making use of the LL model. A promising approach describing rich nonFermi-liquid physics is to consider an anisotropic system as an array of coupled one-dimensional wires [8,9]. Previously, various exotic states were considered in the framework of the sliding Luttinger liquid (sLL) model [10], where the RG analysis of the impact of a single impurity embedded into a LL [11] or continuous disorder in a LL [12] has been generalized for a multichannel case. A subsequent analysis [13] allowing for renormalization of the interaction by disorder has shown that the conducting state does not survive at zero temperature for any realistic long-range interwire interactions. The only quantum phase transition found in Ref. [13] was a superconductor-insulator one with the boundary distorted by disorder.

A single Luttinger liquid cannot describe a metal-insulator transition at high temperatures where quantum interference does not manifest itself. The only phase transition that is known to happen is the Berezinskii-Kosterlitz-Thouless (BKT) one [14], which takes place when the Luttinger parameter $K=3 / 2$ (see Ref. [7]), whereas repulsive electrons correspond to $K<1$. The main advantage of the sLL model is that interwire repulsive interactions stabilize the conducting phase, bringing the condition for the BKT transition into the realm of repulsive fermions. The main disadvantage of the sLL is that it is unstable (for some nonuniversal system parameters) with respect to perturbations like charge-density and Josephson couplings, along with single-particle interwire hybridization. These perturbations may become relevant and destroy the sLL phase at zero temperature.

In this paper, we focus on the phases existing in the presence of continuous disorder at finite temperatures when only the disorder strength and the electron-electron interaction need to be renormalized, generalizing the recently developed method [15] based on the scattering matrix formalism. Since we are not interested in the regime of very low temperatures, where quantum interference governs the transport properties, we may assume that even relevant interwire perturbations do not blow up, provided that their bare values are sufficiently small and temperature infrared cutoff is relatively high. This is the case of an array of wires that are well separated from each other (weak hybridization means weak single- and two-particle Josephson couplings), and the interaction potential between the wires is smooth on the scale of the Fermi wavelength, so that the bare value of interwire charge-density-wave interaction is small. The temperature $T_{*}$, when various interchannel correlations, such as the single-particle hopping, charge-density wave (CDW), and Josephson couplings, may become relevant, is known to be defined by the bare couplings $J_{*}$, the bandwidth $D$, and the scaling dimension $\Delta_{*}$ of the most dangerous hybridization term: $T_{*} \sim D\left(J_{*} / D\right)^{\alpha}$, with the exponent $\alpha=$ $\left(2-\Delta_{*}\right)^{-1}$. In materials with a small overlap between electron wave functions belonging to the adjacent wires, the bare values of the single-particle and correlated-pair interwire (Josephson) hopping are also small. The CDW coupling is proportional to 
the Fourier transform of the Coulomb potential at $2 k_{\mathrm{F}}$, and its bare value is small for a smooth (on the scale of the Fermi wave length) potentials. Under these assumptions, there is a wide temperature range $T \gg T_{*}$, where we may safely use the model of interacting but not hybridized wires (channels).

\section{THE MULTICHANNEL MODEL}

The action describing a multichannel LL is a straightforward generalization of the standard LL action [7]:

$$
\begin{aligned}
S= & \frac{1}{8 \pi} \int d x d t \boldsymbol{\Psi}^{\mathrm{T}}\left[\hat{\tau}_{1} \partial_{t}+\mathrm{V} \partial_{x}\right] \partial_{x} \boldsymbol{\Psi} \\
& +i D \sum_{i} \int d x d t d t^{\prime} \cos \left[\theta_{i}(x, t)-\theta_{i}\left(x, t^{\prime}\right)\right]
\end{aligned}
$$

Here the composite vector field $\boldsymbol{\Psi}^{\mathrm{T}}=\left(\boldsymbol{\theta}^{\mathrm{T}}, \boldsymbol{\phi}^{\mathrm{T}}\right)$ is built on two vector fields, $\boldsymbol{\theta}=\left(\theta_{1}, \ldots, \theta_{N}\right)$ and $\boldsymbol{\phi}=\left(\phi_{1}, \ldots, \phi_{N}\right)$, that parametrize density and current excitations in the $i$ th channel $(1 \leqslant i \leqslant N)$ as $\rho_{i}=\frac{1}{2 \pi} \partial_{x} \theta_{i}$ and $j_{i}=\frac{1}{2 \pi} \partial_{x} \phi_{i} ; \hat{\tau}_{1}$ is the Pauli matrix in $\{\boldsymbol{\theta}, \boldsymbol{\phi}\}$ space, and $\mathrm{V}=\operatorname{diag}\left[\mathrm{V}_{+}, \mathrm{V}_{-}\right]$ is a block-diagonal (in the same space) matrix describing density-density, $\mathrm{V}_{+}$, and current-current, $\mathrm{V}_{-}$, interactions. In the absence of interchannel interactions these matrices would become diagonal, $\left[V_{ \pm}\right]_{i j} \rightarrow \delta_{i j} v_{i} K_{i}^{\mp 1}$, with $v_{i}$ and $K_{i}$ being the velocities and Luttinger parameters in the $i$ th channel.

The nonlinear, cosine term in the action results from the standard replica averaging over disorder, as in the singlechannel case [7], although the replica indices are suppressed in Eq. (1). The averaging has been performed over the standard single-particle disorder potential with random backscattering amplitudes, $\xi_{i}(x) e^{i \theta_{i}(x)}+$ c.c., with the white-noise correlations,

$$
\left\langle\xi_{i}(x) \bar{\xi}_{j}\left(x^{\prime}\right)\right\rangle=\delta_{i j} D_{i} \delta\left(x-x^{\prime}\right),
$$

which is a standard approximation when the correlation length is shorter than the mean free path and interwire distance (longrange correlations will require a separate study).

\section{RG equations}

Following the standard procedure [7], one derives the following RG equations for the disorder strength and interaction matrices (with $l$ being the logarithm of the scaling factor):

$$
\begin{aligned}
\partial_{l} \mathrm{D} & =(3-2 \widetilde{\mathrm{K}}) \mathrm{D}, \quad \mathrm{D}(l=0)=D_{0} \mathbb{1}, \\
\partial_{l} \mathrm{~V}_{-}^{-1} & =\mathrm{D}, \quad \mathrm{V}_{-}(l=0)=\mathrm{V}_{-}^{(0)} .
\end{aligned}
$$

The density-density interaction matrix $V_{+}$does not renormalize: $\partial_{l} \mathrm{~V}_{+}=0$. In Eqs. (3), we have introduced matrices $\mathrm{D}$, and $\widetilde{\mathrm{K}}$ which are diagonal in the channel space: $\mathrm{D}=\operatorname{diag}\left\{D_{1}, \ldots, D_{N}\right\}$ and $\widetilde{\mathrm{K}}=\operatorname{diag}\left\{K_{11}, \ldots, K_{N N}\right\}$. The elements of $\mathrm{D}$ describe the disorder strength in the appropriate channel, Eq. (2), while the elements of $\widetilde{\mathrm{K}}$ are the diagonal elements of the Luttinger matrix $\mathrm{K}$, which is defined from the equation

$$
\mathrm{KV}_{+} \mathrm{K}=\mathrm{V}_{-} \text {. }
$$

In the $N$-channel RG equations, the Luttinger matrix plays a role similar to that of the Luttinger parameter in the singlechannel LL (see Ref. [15] for details). Equation (4) closes the set of RG equations (3). Below we build the phase diagrams corresponding to these equations for two particular cases.

\section{LATTICE OF IDENTICAL CHANNELS}

Here we consider the multichannel model where identical channels (wires) are packed into a two-dimensional (2D) or three-dimensional array in such a way that the cross section perpendicular to the length of the wires forms a lattice $\mathcal{L}$. All matrix elements of the interaction matrices $V_{ \pm}$ may be labeled by the spatial positions $\boldsymbol{R}$ of wires in the perpendicular plane, $\mathrm{V}_{ \pm} \rightarrow V_{ \pm}\left(\boldsymbol{R}, \boldsymbol{R}^{\prime}\right)$, where $\boldsymbol{R} \subset \mathcal{L}$. If the lattice is a Bravais one, the matrix elements of $V_{ \pm}$become scalars, $V_{ \pm}\left(\boldsymbol{R}-\boldsymbol{R}^{\prime}\right)$ (assuming translation invariance and periodic boundary conditions). For non-Bravais lattices they will become matrices in the space of inequivalent wires, which we do not consider here. Equation (4) for the Luttinger matrix transforms to

$$
\sum_{\boldsymbol{R}_{1}, \boldsymbol{R}_{2} \subset \mathcal{L}} K\left(\boldsymbol{R}_{12}\right) V_{+}\left(\boldsymbol{R}_{23}\right) K\left(\boldsymbol{R}_{34}\right)=V_{-}\left(\boldsymbol{R}_{14}\right),
$$

where $\boldsymbol{R}_{i j}=\boldsymbol{R}_{i}-\boldsymbol{R}_{j}$. This equation is easily solved with the use of the discrete Fourier transform on the lattice $\mathcal{L}$,

$$
F(\boldsymbol{R})=\int \frac{d^{d} q}{(2 \pi)^{d}} F(\boldsymbol{q}) e^{i \boldsymbol{q} \boldsymbol{R}}, \quad F(\boldsymbol{q})=\sum_{\boldsymbol{R} \subset \mathcal{L}} F(\boldsymbol{R}) e^{-i \boldsymbol{q} \boldsymbol{R}} .
$$

Here and elsewhere in this section the momentum integration is performed over the Brillouin zone of the wire lattice. The solution to Eq. (5) has the form

$$
K(\boldsymbol{r})=\int \frac{d^{d} q}{(2 \pi)^{d}} \sqrt{\frac{V_{-}(\boldsymbol{q})}{V_{+}(\boldsymbol{q})}} e^{i \boldsymbol{q} \boldsymbol{r}},
$$

with $\boldsymbol{r}=\boldsymbol{R}-\boldsymbol{R}^{\prime} \subset \mathcal{L}$ and $V_{ \pm}(\boldsymbol{q})$ being the discrete Fourier transform of interaction potentials $V_{ \pm}(\boldsymbol{r})$.

Now the diagonal matrix $\widetilde{\mathrm{K}}$ in Eqs. (3) is reduced to $\widetilde{K} \mathbb{1}$, where the effective Luttinger parameter $\widetilde{K}$ is given by $\widetilde{K} \equiv$ $K(\boldsymbol{r}=\mathbf{0})$. Assuming equal strength of bare disorder in each wire, $D_{i}=D$, the matrix RG equations (3) are reduced to the two RG equations for the disorder strength and the deviation $c$ of the Fourier transform of the current-current interaction from its bare value:

$$
\begin{aligned}
\partial_{l} D & =[3-2 \widetilde{K}(c)] D, \quad \partial_{l} c=D, \\
D(l=0) & =D_{0}, \quad c(l=0)=0,
\end{aligned}
$$

with $c(l)$ defined by

$$
c(l) \equiv V_{-}^{-1}(\boldsymbol{q} ; l)-V_{0}^{-1}(\boldsymbol{q}),
$$

where $V_{0}(\boldsymbol{q}) \equiv V_{-}(\boldsymbol{q}, l=0)$ is the bare value of the currentcurrent interaction. The closure to the $\mathrm{RG}$ equations is provided by the explicit dependence of $\widetilde{K} \equiv \widetilde{K}(\boldsymbol{r}=\mathbf{0})$ on $c$ :

$$
\widetilde{K}(c)=\int \frac{d^{d} q}{(2 \pi)^{d}} V_{+}^{-1 / 2}(\boldsymbol{q})\left[V_{0}(\boldsymbol{q})+c(l)\right]^{-1 / 2} .
$$

The effective Luttinger parameter $\widetilde{K}(c)$ is a monotonically decreasing function of $c$, and therefore, if its bare value $\widetilde{K}_{0} \equiv \widetilde{K}(c=0)<3 / 2$, the disorder will always grow under renormalization, and we always end up in the insulating 


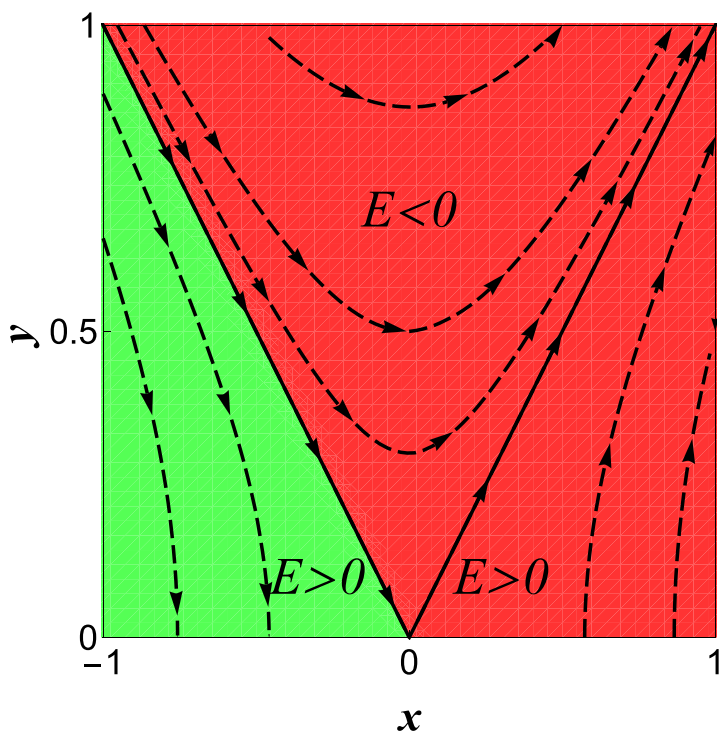

FIG. 1. BKT flow diagram for the wire lattice, with $y=\sqrt{\kappa D}$ characterizing the disorder and $x$ being the deviation from the critical value of the Luttinger parameter $\widetilde{K}_{0}=\frac{3}{2}$. The equations have a conserved "energy" $E$. If $E<0$ or $E>0$ and $x>0$, the system flows to strong disorder, while only if $E>0$ and $x<0$ does it flow to a conducting state.

regime. From now on, we will be interested only in the case $\widetilde{K}_{0}>3 / 2$.

The BKT transition takes place at $c=c^{*}$, with the critical value $c^{*}$ found from $\widetilde{K}\left(c^{*}\right)=\frac{3}{2}$. The analysis of the RG flow in these terms is possible only in the vicinity of the BKT critical value $\widetilde{K}=3 / 2$. This means that the bare value $\widetilde{K}_{0}$ should also be close to $3 / 2$. In this case $c^{*} \ll 1$, and we may approximate

$$
\widetilde{K}(c) \approx \widetilde{K}_{0}-\kappa c,
$$

where

$$
\kappa=-\left.\frac{d \tilde{K}(c)}{d c}\right|_{c=0}=\frac{1}{2} \int \frac{d^{d} q}{(2 \pi)^{d}} \frac{V_{0}^{3 / 2}(\boldsymbol{q})}{V_{+}^{1 / 2}(\boldsymbol{q})} .
$$

The critical value $c^{*}$ is given in terms of the initial detuning from the transition:

$$
c^{*}=\frac{\delta}{\kappa} \ll 1, \quad \delta \equiv \widetilde{K}_{0}-\frac{3}{2} \geqslant 0 .
$$

The BKT RG equations acquire the standard form,

$$
\partial_{l} D=2 x D, \quad \partial_{l} x=\kappa D, \quad x \equiv \kappa c-\delta,
$$

with the initial condition $x(l=0)=-\delta$ in terms of $x$. The RG flows in the $(x, D)$ plane obey the equation

$$
\kappa \frac{d D}{d x}=2 x,
$$

which defines the family of trajectories $D=x^{2} \kappa^{-1}+E$, with the constant $E$ being defined by the initial values, $E=D_{0}-$ $\delta^{2} \kappa^{-1}$.

The boundary between the insulating and conducting phases corresponds to $E=0$, i.e., $\delta=\sqrt{\kappa D_{0}} \equiv y$ : the system is conducting for $\delta>\sqrt{\kappa D_{0}}$ and insulating for $\delta<\sqrt{\kappa D_{0}}$. These RG flows are illustrated in Fig. 1, where the phase boundary is clearly seen. The effects of interwire interactions are in the definitions of the parameters, given explicitly in Eqs. (9)-(11).

The position of the phase-separation boundary is mainly dictated by the interaction, $\widetilde{K}_{0}=\frac{3}{2}$, which also governs renormalization of the (weak) disorder strength while the feedback from disorder to interaction is negligible. One can show [16] that the interwire long-range interaction results in $\widetilde{K}_{0}>K$ (where $K$ is the standard single-wire Luttinger parameter), so that it favors a conducting state. The effective Luttinger parameter $\widetilde{K}_{0}$ depends on both $K$ and interwire interaction parameters and can reach the value $3 / 2$ even for $K<1$ corresponding to repulsive fermions. Therefore, one should expect a competition between the weak interwire long-range interaction and weak disorder leading to the BKT metal-insulator transition for the wire lattice.

\section{TWO DISTINCT CHANNELS}

We limit our analysis of nonidentical channels with different (and uncorrelated) disorder strengths to the case of two channels. Then all matrices in the RG equations (3) are $2 \times 2$. Like in the approach used in the previous section for the $N$-channel problem, we now introduce two renormalizable scalars $c_{1,2}$ describing the deviation of the current-current interaction matrix from its bare value, $\mathrm{V}_{0} \equiv \mathrm{V}_{-}(l=0)$ :

$$
\mathrm{V}_{-}^{-1}(l)=\mathrm{V}_{0}^{-1}+\mathrm{c}, \quad \mathrm{c}=\left(\begin{array}{cc}
c_{1} & 0 \\
0 & c_{2}
\end{array}\right) \text {. }
$$

The RG equations (3) with the new variables become

$$
\begin{aligned}
\partial_{l} \mathrm{D} & =[3-2 \widetilde{\mathrm{K}}(\mathrm{c})] \mathrm{D}, \quad \mathrm{D}(l=0)=D_{0} \mathbb{1}, \\
\partial_{l} \mathrm{C} & =\mathrm{D}, \quad \mathrm{c}(l=0)=0 .
\end{aligned}
$$

Again, $\widetilde{\mathrm{K}}=\operatorname{diag}\left\{K_{11}, K_{22}\right\}$, with the two effective Luttinger parameters $K_{i i}\left(c_{1}, c_{2}\right)$ being the diagonal elements of the Luttinger matrix K, Eq. (4). This equation can now be rewritten via $\mathrm{V}_{0}$ and $\mathrm{c}$ as

$$
\mathrm{KV}_{+} \mathrm{K}\left[\mathrm{V}_{0}^{-1}+\mathrm{c}\right]=\mathbb{1}
$$

Assuming the system to be initially in the vicinity of a generalized BKT transition in each channel (i.e., for both $i=$ 1,2 one has $\left.\left|K_{i i}^{(0)}-3 / 2\right| \ll 1\right)$, one can see that the critical values at which the BKT transition occurs,

$$
K_{i i}\left(c_{1}^{*}, c_{2}^{*}\right)=\frac{3}{2}, \quad i=1,2,
$$

are small, $\left|c_{i}^{*}\right| \ll 1$. Therefore, in the vicinity of the transition one may use the expansion

$$
K_{i i}\left(c_{1}, c_{2}\right) \approx K_{i i}^{(0)}-\sum_{j} \kappa_{i j} c_{j}, \quad \kappa_{i j} \equiv-\left.\frac{\partial K_{i i}}{\partial c_{j}}\right|_{c_{1}=c_{2}=0} .
$$

The matrix of derivatives $\left\{\kappa_{i j}\right\}$ is a symmetric positively definite matrix with the positive matrix elements

$$
\begin{aligned}
\kappa_{i i} & =T^{-1}\left[\left(1-k^{2}\right) V_{i i}^{2}+\operatorname{det} \mathrm{V}\right] \geqslant 0, \\
\kappa & =T^{-1}\left[\left(1-k^{2}\right) V_{12}^{2}+k^{2} \operatorname{det} \mathrm{V}\right] \geqslant 0,
\end{aligned}
$$


where $\kappa \equiv \kappa_{12}=\kappa_{21}$ (see Appendix A for the derivation).

The RG equations (16) turn into

$$
\begin{gathered}
\partial_{l} D_{i}=2 x_{i} D_{i}, \quad \partial_{l} x_{i}=\sum_{j=1,2} \kappa_{i j} D_{j}, \\
D_{i}(l=0)=D_{0}, \quad x_{i}(l=0)=-\delta_{i},
\end{gathered}
$$

where we have introduced the notations

$$
x_{i}=\sum_{j} \kappa_{i j} c_{j}-\delta_{i}, \quad \delta_{i}=K_{i i}^{(0)}-\frac{3}{2}
$$

to present them in the form familiar from the previous section. The substitution $D_{i}=y_{i}^{2}$ reduces Eqs. (21) to the pair of coupled BKT equations:

$$
\begin{aligned}
\partial_{l} y_{i} & =x_{i} y_{i}, \quad \partial_{l} x_{i}=\sum_{j} \kappa_{i j} y_{j}^{2}, \\
D_{i}(l=0) & =D_{0}, \quad x_{i}(l=0)=-\delta_{i} .
\end{aligned}
$$

There is only one integral of motion (see Appendix B for details), given in terms of $\boldsymbol{x}=\left(x_{1}, x_{2}\right)$ and $\boldsymbol{y}=\left(y_{1}, y_{2}\right)$ by

$$
E=\boldsymbol{x} \mathrm{m} \boldsymbol{x}-\boldsymbol{y}^{2}, \quad \mathrm{~m}=\hat{\kappa}^{-1}=\left(\begin{array}{cc}
m_{1} & -m \\
-m & m_{2}
\end{array}\right),
$$

where $m_{1,2}$ and $m$ are positive.

In the absence of the interchannel coupling, $m=0$, Eqs. (23) would describe two independent systems undergoing the BKT transition. They are equivalent to two uncoupled Kondo impurities,

$$
\partial_{l} y_{i}=x_{i} y_{i}, \quad \partial_{l} x_{i}=\kappa_{i i} y_{i}^{2},
$$

each having the integral of motion ("energy"),

$$
E_{i}=m_{i} x_{i}^{2}-y_{i}^{2},
$$

with the exchange constants $J_{i}^{\perp} \equiv y_{i}$ and $J_{i}^{\|} \equiv x_{i}$. Then $\delta_{i}<0$ in Eqs. (21) corresponds to the antiferromagnetic Kondo impurity $\left(J_{i}^{\|}>0\right)$ where all the RG flows go towards the strong-coupling fixed point (the unitary limit of the Kondo screening when $J_{i}^{\perp} \rightarrow \infty$ at the Kondo temperature) corresponding to the insulator. The case of $\delta_{i}>0$ corresponds to the ferromagnetic Kondo impurity, $J_{i}^{\|}<0$, where the flows go to the strong-coupling fixed point for $E_{i}<0$ but to the weak-coupling fixed point corresponding to the conductor for $E_{i}>0$. For completeness, these well-known results, including explicit expressions for the RG flows, are recouped in Appendix B.

Let us analyze an impact of a weak interchannel coupling, $m \ll 1$, when Eqs. (23) describe two coupled Kondo impurities. In this case, there is only one integral of motion, Eqs. (24), while the former integrals of motion, Eq. (25), become "adiabatic invariants," i.e., slow functions of "time" $l$ :

$$
\dot{E}_{i}=2 m x_{i} \dot{x}_{-i} \text {. }
$$

In Appendix $\mathrm{C}$, we show how to use these invariants to construct the RG flows starting from the uncoupled case. Here we summarize the results of this consideration. In what follows, we will use the abbreviations to refer to different phases. The phases where both channels are either conducting or insulating will be denoted as $(c c)$ and $(i i)$, respectively. The phase where one of the channels is conducting and another is insulating will be referred to as either $(c i)$ or $(i c)$ depending on THE details, i.e., which channel is blocked and which is not.

If both $\delta_{i} \leqslant 0$ in Eqs. (21), the system flows to the strongcoupling Kondo regime, i.e., an insulator. This means that the (ii) phase where both channels were insulators for $m=0$ is not qualitatively affected by the coupling between channels but just expanding in the phase space.

If both $\delta_{i} \geqslant 0$, the RG flows depend on the bare values of the adiabatic invariants $E_{i}(0)$. They start with a negative derivative, Eq. (26), bending upwards in comparison to those without the coupling. The flows are illustrated in Fig. 2, where the trajectories are numerically calculated for several values of the parameters. Note that the critical value for the initial values of $E_{i}$ to stay on a conducting trajectory [leading to $y_{i}(l=\infty)=0$ ] increases so that the $(c c)$ phase shrinks.

The mixed $(c i)$ phase turns out to be totally unstable, as illustrated in Fig. 2. Since the RG trajectories in the insulating channel flow towards the strong-coupling Kondo regime, the negative-energy shift in the initially conducting channel arising from the coupling will be sufficient to drag the channel into the negative-energy region (see Appendix C for details), finally making it insulating as well. Thus, the intermediate $c i$ phase eventually disappears due to the interchannel coupling, while the BKT transition between the (ii) and ( $c c$ ) phases is shifted towards the insulator.

\section{CONCLUSION}

We have constructed a generic description of a "high"temperature Berezinskii-Kosterlitz-Thouless transition in a multichannel array of coupled Luttinger liquids. We have focused on two cases, a lattice of identical LL wires and two distinct LL channels. The interchannel coupling makes these transitions, in principle, observable not only in systems with locally repulsive bosons but also in systems with repulsive fermions, where no such transition exists for a single LL channel.

\section{ACKNOWLEDGMENTS}

I.V.Y. gratefully acknowledges support from the Leverhulme Trust via Grant No. RPG-2016-044 and hospitality extended to him at the Center for Theoretical Physics of Complex Systems, Daejeon, South Korea.

\section{APPENDIX A: CALCULATION OF $\kappa_{i j}$, THE MATRIX OF DERIVATIVES}

Instead of differentiating the explicit solution with respect to $c$ at the BKT transition, it is easier to start from linearizing Eq. (17) in $\mathrm{c}$, using $\mathrm{K}(\mathrm{c})=\mathrm{K}+\delta \mathrm{K}$, where the diagonal elements of $\mathrm{K} \equiv \mathrm{K}(l=0)$ are taken at the BKT transition $K_{i i}=3 / 2$ :

$$
\mathrm{K}=\frac{3}{2}\left(\begin{array}{ll}
1 & k \\
k & 1
\end{array}\right)
$$




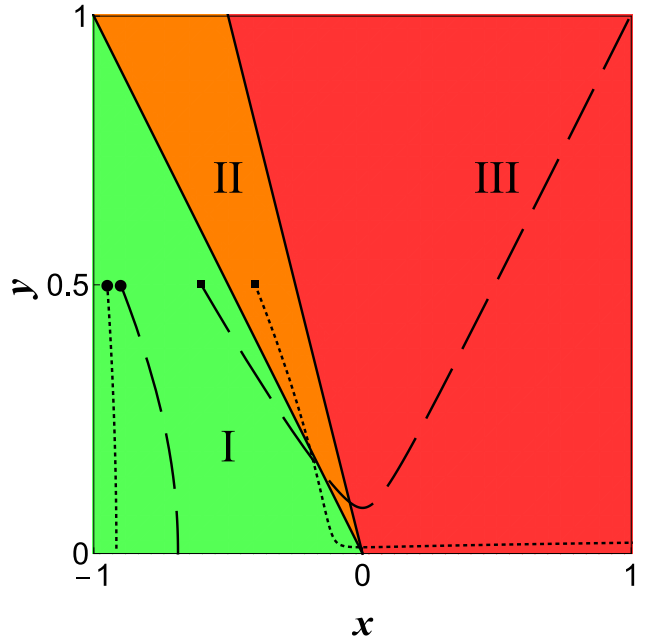

(a)

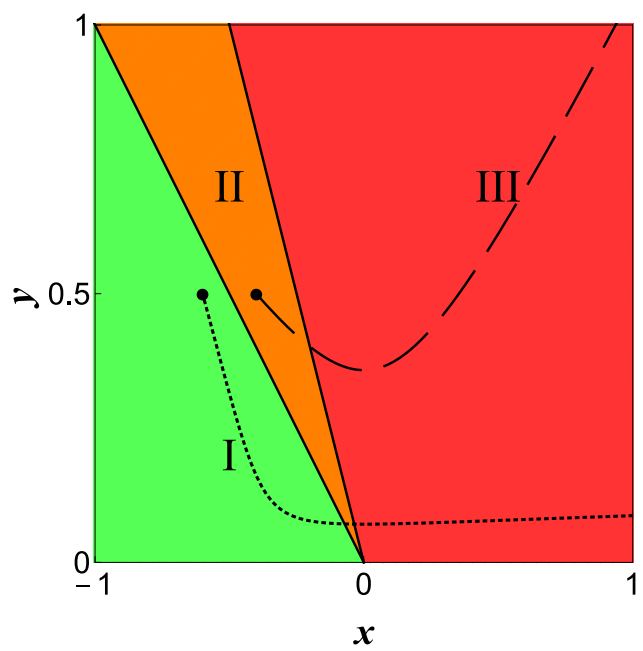

(b)

FIG. 2. Phase diagram of a two-channel disordered Luttinger liquid. Differently shaded regions I (green), II (amber), and III (red) correspond to three phases of the uncoupled channels: $c c$ (I), $i c$ (II), and $i i$ (III). Three pairs of trajectories that show the effect of the interchannel coupling $\kappa$ were calculated for $\kappa=0.4 \kappa_{11}, \kappa_{22}=0.25 \kappa_{11}$ in Eqs. (20) and $y_{0}=\left(4 \kappa_{11}\right)^{-1 / 2}$ in Eqs. (23), with $\kappa_{11}$ defining the scale. In each pair, the dashed and dotted lines correspond to channels 1 and 2, respectively. The pair started with circles in (a) corresponds to a system with energy $E \approx 2.1 \kappa_{11}$ deep inside the ( $c c$ ) region where the coupling does not lead to qualitative changes. The pair started with squares in (a) corresponds to $E \approx 0.07 \kappa_{11}$, which is ostensibly in the $(c c)$ region for an uncoupled system; the coupling generates a large enough negative-energy shift to push the system into the (ii) region. The pair in (b) is for $E \approx 0.91 \kappa_{11}$ when the dashed trajectory is in the insulating region of channel 1 . As the disorder there begins to grow, the dotted trajectory for channel 2 that remains in the conducting region for $\kappa=0$ is dragged into the insulating region with it. This shows how the mixed (ic) phase is destroyed by interchannel coupling.

Here the only restriction on the off-diagonal element of $\mathrm{K}$ is $|k| \leqslant 1$. Using Eq. (17) at the BKT point allows us to eliminate $\mathrm{V}_{+}$:

$$
\mathrm{V}_{+}=\mathrm{K}^{-1} \mathrm{~V}_{0} \mathrm{~K}^{-1}
$$

Then the linearization results in

$$
\mathrm{V}_{0}^{-1} \delta \mathrm{KK}^{-1}+\text { transposition }=-\mathrm{c} .
$$

This equation has a solution parametrized by an arbitrary scalar $\omega$,

$$
\delta \mathrm{K}=-\frac{1}{2} \mathrm{~V}_{0}\left[\mathrm{c}+\omega \hat{\sigma}_{2}\right] \mathrm{K},
$$

where $\hat{\sigma}_{2}=i \hat{\tau}_{2}$ and $\hat{\tau}_{2}$ is a Pauli matrix. The scalar $\omega$ is found from the fact that both $\mathrm{K}$ and $\delta \mathrm{K}$ are symmetric matrices. This condition can be written as $\operatorname{tr} \hat{\sigma}_{2} \delta \mathrm{K}=0$, which results in

$$
\begin{aligned}
& \omega=-T^{-1} \operatorname{tr} \mathrm{K} \hat{\sigma}_{2} \mathrm{~V}_{0} \mathrm{c}, \\
& T=-\operatorname{tr} \mathrm{K} \hat{\sigma}_{2} \mathrm{~V}_{0} \hat{\sigma}_{2}=V_{11}+V_{22}-2 k V_{12} \geqslant 0,
\end{aligned}
$$

with $V_{i j}$ being the matrix elements of $\mathrm{V}_{0}$. Substituting this into Eq. (A2) and differentiating $\delta \mathrm{K}$ with respect to $c_{i}$ gives (up to a factor) the matrix $\kappa_{i j}$, Eqs. (19):

$$
\kappa_{i j}=K_{i j} V_{j i}-T^{-1}\left(\mathrm{~K} \hat{\sigma}_{2} \mathrm{~V}\right)_{i i}\left(\mathrm{~K} \hat{\sigma}_{2} \mathrm{~V}\right)_{j j} .
$$

The matrix $\hat{\kappa}$ is symmetric with positive matrix elements. One can easily check that $\operatorname{det} \hat{\kappa} \geqslant 0$ so that both $\hat{\kappa}$ and $\hat{\kappa}^{-1}$ are positive-definite matrices.

\section{APPENDIX B: INTEGRALS OF MOTION AND RG FLOWS}

Equations (23) define the RG flows in four-dimensional space $\left\{y_{i}, x_{i}\right\}$, with $i=1,2$. Since we are interested in only the asymptotic behavior of $D_{i} \equiv y_{i}^{2}$, we eliminate $x_{i}$ using the first of Eqs. (23) to obtain $x_{i}=\partial_{l} \ln y_{i}$. Introducing the "force" $F_{i} \equiv D_{i}=y_{i}^{2}$, denoting $\partial_{l} q_{i} \equiv \dot{q}_{i}$, introducing the "mass tensor" $\mathrm{m}$ with positive $m_{1,2}$ and $m$, and parametrizing $y_{i}$ as

$$
y_{i}=e^{q_{i}}, \quad \mathrm{~m}=\hat{\kappa}^{-1} \equiv\left(\begin{array}{cc}
m_{1} & -m \\
-m & m_{2}
\end{array}\right),
$$

we cast the second of Eqs. (23) into the equations of motion of a Newtonian particle in a 2D space,

$$
\mathrm{m} \ddot{\boldsymbol{q}}=\boldsymbol{F}(\boldsymbol{q}),
$$

with the initial conditions $q_{i}(0)=\frac{1}{2} \ln D_{0}$ and $\dot{q}_{i}(0)=-\delta_{i}$. The corresponding Lagrangian is

$$
L=\frac{1}{2} \dot{\boldsymbol{q}} \mathrm{m} \dot{\boldsymbol{q}}-U(\boldsymbol{q}), \quad U(\boldsymbol{q})=-\frac{1}{2} \sum_{i} e^{2 q_{i}} .
$$

Then the total energy, $E=\frac{1}{2} \dot{\boldsymbol{q}} \mathrm{m}+U(\boldsymbol{q})$, is the integral of motion,

$$
E=\frac{1}{2} \dot{\boldsymbol{q}} \mathrm{m} \dot{\boldsymbol{q}}-\frac{1}{2} \sum_{i} e^{2 q_{i}}
$$

given in terms of $\boldsymbol{x}$ and $\boldsymbol{y}$ in the main text, Eqs. (24).

If the mass tensor is diagonal, $\mathrm{m}=\operatorname{diag}\left[m_{1}, m_{2}\right]$, the Lagrangian would be a sum of two Lagrangians, $L=\sum_{i} L_{i}$, given by

$$
L_{i}=\frac{1}{2} m_{i} \dot{q}_{2}^{2}+\frac{1}{2} e^{2 q_{i}} .
$$


The explicit solution of the equations of motion,

$$
D_{i}=e^{2 q_{i}}=\frac{m_{i} k_{i}^{2}}{\sinh ^{2}\left[k_{i} l+\chi_{i}\right]},
$$

depends on the following parameters found from the initial conditions:

$$
k_{i}^{2}=\delta_{i}^{2}-\frac{D_{0}}{m_{i}}, \quad \tanh \chi_{i}=\frac{k_{i}}{\delta_{i}} .
$$

This solution has the energy $E_{i}=\frac{1}{2} m_{i} k_{i}^{2}$. For positive energy the positive branch of the square root is assumed, $k_{i}=\sqrt{2 E_{i} / m_{i}}$. As one can see, for the positive energy the disorder flows to zero (conducting phase):

$$
D_{i}(l \rightarrow \infty) \rightarrow 0, \quad E_{i}=\frac{1}{2}\left[\frac{\delta_{i}^{2}}{m_{i}}-D_{0}\right]>0 .
$$

The same solution, Eq. (B5), is applicable for negative energies, $E_{i} \rightarrow-\left|E_{i}\right|$ and $k_{i} \rightarrow i\left|k_{i}\right|=i \sqrt{2\left|E_{i}\right| / m_{i}}$ :

$$
D_{i}=e^{2 q_{i}}=\frac{m_{i}\left|k_{i}\right|^{2}}{\sin ^{2}\left[\left|k_{i}\right| l+\chi_{i}\right]},
$$

with the necessary change $\chi_{i} \rightarrow i \chi_{i}$ to satisfy the initial conditions,

$$
-\left|k_{i}\right|^{2}=\delta_{i}^{2}-\frac{D_{0}}{m_{i}}, \quad \tan \chi_{i}=\frac{\left|k_{i}\right|}{\delta_{i}} .
$$

This is the solution for insulating behavior that demonstrates divergence at a finite "length" $l_{\mathrm{K}}$, corresponding to the finite Kondo temperature. Without coupling between channels, we have a 2D Newtonian particle moving in a potential that is a sum of two parts, each depending upon only one coordinate. The degrees of freedom decouple, and we have two uncoupled equations with two integrals of motion (energies for two directions), one for each equation.

\section{APPENDIX C: RG FLOWS FOR TWO COUPLED CHANNELS}

The coupling between channels introduces anisotropy in the dispersion law that couples motions in two perpendicular directions, which means that $E_{1}$ and $E_{2}$ are no longer integrals of motion. Now we have a single integral of motion, the total energy, Eqs. (24). Nevertheless, for the weak coupling ( $m \ll$ 1) we may apply adiabatic perturbation theory using the fact that both $E_{i}$ will now be slowly changing:

$$
\dot{E}_{i}=m \dot{q}_{i} \ddot{q}_{-i} \text {. }
$$

Here we have used the convention $-1 \rightarrow 2,-2 \rightarrow 1$ for the $-i$ index above. Since the energy is adiabatically changing, we can write approximate expressions for the solution, similar to Eq. (B5):

$$
\begin{gathered}
D_{i}(l)=e^{2 q_{i}(l)}=\frac{m_{i} k_{i}^{2}(l)}{\sinh ^{2} \theta_{i}(l)}, \\
\theta_{i}(l)=\int_{0}^{l} d l^{\prime} k_{i}\left(l^{\prime}\right)+\chi_{i} .
\end{gathered}
$$

Which solution to use, conducting or insulating, depends on the energies $E_{i}=m_{i} k_{i}^{2} / 2$. As they are not conserved due to the weak coupling, we could start from the energy of one sign (e.g., positive, corresponding to the conducting behavior), but the correction could bring us into the negative territory where we have to switch to the insulating solution, as illustrated in Fig. 2.

The adiabatic change of $E_{1,2}$ is governed by Eq. (C1), which already contains the small parameter $m$, so that we can use the uncoupled solutions from Eqs. (B5) and (B7) there. It is important to stress that $\ddot{q}_{i} \geqslant 0$ for both the solutions and $\dot{q}_{i} \leqslant 0$ for the conducting solution, while for the insulating solution it is positive if $\delta_{i} \leqslant 0$ and starts from negative for $\delta_{i} \geqslant 0$ but later turns positive.

These equations are not applicable in the strong-coupling limit, where they give only a rough idea of the relevance of the Kondo physics: for $\dot{q}_{i}>0$ the system flows towards the strong-coupling Kondo regime corresponding to an insulator. We therefore focus on the dynamics in the region with a starting point $x_{i} \equiv \dot{q}_{i}<0$, corresponding to $\delta_{i}>0$ in Eqs. (21).

When both channels are initially in either conducting, $(c c)$, or insulating, (ii), phases, a rough estimate for the correction to the energies gives

$$
\delta E_{i}(l)=\frac{m}{m_{i}}\left[D_{i}(l)-D_{0}\right] .
$$

Then in the $(c c)$ phase, where $D_{i}(l \rightarrow \infty) \rightarrow 0$, both initially positive energies acquire finite negative corrections,

$$
\delta E_{i}=-\frac{m}{m_{i}} D_{0}=\frac{m_{i}}{2}\left[\delta_{i}^{2}-\left(\frac{1}{m_{i}}+\frac{m}{m_{i}^{2}}\right) D_{0}\right],
$$

which simply shifts the boundary of stability further into the region of higher Luttinger parameters $K_{i i}$ so that the (cc) phase shrinks. In the ( $i$ i ) phase we can still use Eq. (C3) until both $D_{i}$ reach their minimum values (similar to the nonmonotonic dependence of $J_{\perp}$ in the ferromagnetic Kondo system). Thus, a negative energy acquires a negative correction, so that there is no qualitative change: the $(i i)$ phase remains itself.

In contrast to the two previous cases, the interchannel interaction plays a crucial role for the initially mixed (ic) phase, where one channel is conducting and one is insulating. In the region $\dot{q}<0$, we see that the positive energy of the conducting channel is suppressed by the correction, while the negative energy of the insulating channel is boosted further into the negative territory. As before, the interchannel coupling favors insulating behavior and shrinks the $(c i)$ phase.

If we look for qualitative clues into the regime where $\dot{q}>0$ and disorder is growing, Eqs. (B7) and (C2), we see that when approaching the strong-coupling regime (the Kondo temperature) in the insulating channel, the correction to the conducting channel energy blows up. The conducting channel is thus blocked, while the insulating one is strengthened, so that the system moves towards the (ii) phase analyzed above and found to be stable.

To conclude, the mixed ( $c i$ ) phase is destroyed as the Kondo temperature is lowered, while the $(c c)$ and $(i i)$ phase boundaries are shifted in favor of the insulator. Note that although interchannel interactions force the Luttinger matrix to take higher values in order to arrive at a conducting state, the original Luttinger parameters can still be well below the threshold $K>3 / 2$ in an isolated channel. 
[1] P. W. Anderson, Phys. Rev. 109, 1492 (1958).

[2] E. Abrahams, P. W. Anderson, D. C. Licciardello, and T. V. Ramakrishnan, Phys. Rev. Lett. 42, 673 (1979).

[3] L. P. Gor'kov, A. I. Larkin, and D. E. Khmel'nitskii, JETP Lett. 30, 228 (1979).

[4] B. L. Altshuler, A. G. Aronov, and P. A. Lee, Phys. Rev. Lett. 44, 1288 (1980).

[5] D. M. Basko, I. L. Aleiner, and B. L. Altshuler, Ann. Phys. (NY) 321, 1126 (2006); see also [17].

[6] E. Abrahams, S. V. Kravchenko, and M. P. Sarachik, Rev. Mod. Phys. 73, 251 (2001).

[7] T. Giamarchi, Quantum Physics in One Dimension (Clarendon, London, 2004).

[8] J. C. Y. Teo and C. L. Kane, Phys. Rev. B 89, 085101 (2014).

[9] P.-H. Huang, J.-H. Chen, P. R. S. Gomes, T. Neupert, C. Chamon, and C. Mudry, Phys. Rev. B 93, 205123 (2016).

[10] S. L. Sondhi and K. Yang, Phys. Rev. B 63, 054430 (2001); A. Vishwanath and D. Carpentier, Phys. Rev. Lett. 86, 676 (2001); R. Mukhopadhyay, C. L. Kane, and T. C. Lubensky, Phys. Rev. B 63, 081103 (2001); C. L. Kane, R. Mukhopad- hyay, and T. C. Lubensky, Phys. Rev. Lett. 88, 036401 (2002).

[11] C. L. Kane and M. P. A. Fisher, Phys. Rev. Lett. 68, 1220 (1992); Phys. Rev. B 46, 15233 (1992); A. Furusaki and N. Nagaosa, ibid. 47, 4631 (1993).

[12] T. Giamarchi and H. J. Schulz, Phys. Rev. B 37, 325 (1988).

[13] S. Scheidl, S. Bogner, and T. Emig, Phys. Rev. B 65, 224507 (2002).

[14] V. L. Berezinskii, Sov. Phys. JETP 32, 493 (1971); 34, 610 (1972); J. M. Kosterlitz and D. J. Thouless, J. Phys. C 6, 1181 (1973).

[15] I. V. Yurkevich, Europhys. Lett. 104, 37004 (2013); Sci. Rep. 7, 3550 (2017); I. V. Yurkevich, A. Galda, O. M. Yevtushenko, and I. V. Lerner, Phys. Rev. Lett. 110, 136405 (2013); V. Kagalovsky, I. V. Lerner, and I. V. Yurkevich, Phys. Rev. B 95, 205122 (2017).

[16] A. L. Chudnovskiy, V. Kagalovsky, and I. V. Yurkevich, Phys. Rev. B 96, 165111 (2017).

[17] I. V. Gornyi, A. D. Mirlin, and D. G. Polyakov, Phys. Rev. Lett. 95, 206603 (2005). 